\title{
Stage I Rectal Neuroendocrine Tumor AJCC v8
}

National Cancer Institute

\section{Source}

National Cancer Institute. Stage I Rectal Neuroendocrine Tumor A/CC v8. NCI Thesaurus. Code C135528.

Stage I includes: T1, N0, M0. T1: Tumor invading the lamina propria or submucosa and measuring $2 \mathrm{~cm}$ or less. N0: No regional lymph node metastasis. M0: No distant metastasis. (AJCC 8th ed.) 